\title{
Mirrorless Optical Parametric Oscillation with Tunable Threshold in Cold Atoms
}

\author{
Yefeng Mei, ${ }^{1}$ Xianxin Guo, ${ }^{1}$ Luwei Zhao, ${ }^{1,2}$ and Shengwang $\mathrm{Du}^{1, *}$ \\ ${ }^{1}$ Department of Physics, The Hong Kong University of Science and Technology, \\ Clear Water Bay, Kowloon, Hong Kong, China \\ ${ }^{2}$ Light Innovation Technology Limited, Hong Kong, China
}

(Received 5 July 2017; revised manuscript received 27 August 2017; published 13 October 2017)

\begin{abstract}
We report the demonstration of a mirrorless optical parametric oscillator with a tunable threshold in lasercooled atoms with four-wave mixing (FWM) using electromagnetically induced transparency. Driven by two classical laser beams, the generated Stokes and anti-Stokes fields counterpropagate and build up efficient intrinsic feedback through the nonlinear FWM process. This feedback does not involve any cavity or spatially distributed microstructures. We observe the transition of photon correlation properties from the biphoton quantum regime (below the threshold) to the oscillation regime (above the threshold). The pump threshold can be tuned by varying the operating parameters. We achieve the oscillation with a threshold as low as $15 \mu \mathrm{W}$.
\end{abstract}

DOI: 10.1103/PhysRevLett.119.150406

As coherent light sources, mirrorless laser oscillators have some unique properties such as cavity-free alignment and large tunability. Unlike photonic crystal lasers [1,2] and random lasers $[3,4]$ that rely on spatially distributed feedback from ordered or disordered scattering inside the medium, the mirrorless optical parametric oscillation (MLOPO) is based upon nonlinear interaction of counterpropagating fields. Since first proposed 51 years ago [5], MLOPO has been often used for studying the instability and self-oscillation of degenerate four-wave mixing (FWM) in atomic vapor cells [6-10]. In 1999, MLOPO of nondegenerate FWM was realized in a hot rubidium vapor cell with electromagnetically induced transparency (EIT) [11]. Then not until 2007, the first MLOPO for parametric downconversion $\left(\chi^{(2)}\right.$ nonlinearity) was achieved making use of quasiphase matching for realizing the counterpropagation condition [12].

For backward FWM, the phase matching of the $\chi^{(3)}$ nonlinear process endows the possibility of a counterpropagation configuration, but so far FWM-based MLOPO has been achieved only in hot atomic vapor cells, because a large parametric gain requires a high atomic optical depth (OD) [6-8,11]. Although spontaneous FWM with cold atoms having negligible Doppler broadening has been intensively used for generating narrow-band entangled photon pairs (termed biphotons) in the small parametric gain regime [13-15], MLOPO above the threshold has never been achieved. Reference [2] reported OPO in cold atoms, but its spatially distributed feedback is formed from a one-dimensional lattice structure [16], so it is not in the category of MLOPO discussed here with intrinsic feedback purely from the nonlinearity. A similar result has also been obtained with self-organized atomic density grating [17]. In addition, although the theory predicted that by making use of EIT the pump threshold can be tuned [18], this threshold tunability has not been fully exploited experimentally.
In this Letter, we report the demonstration of MLOPO with a tunable threshold in laser-cooled atoms. Without involving any cavity or spatially distributed linear scatterers, the intrinsic feedback is built up by the counterpropagating nonlinear FWM process with EIT. Unlike the periodically poled $\chi^{(2)}$ nonlinear crystal whose quasiphase-matching condition and fixed geometry are extremely difficult to be tuned in situ, our atomic-system-based FWM provides a large tunability as demonstrated in this work. As compared to the hot atomic vapor systems in which the single-photon quantum level is difficult to reach because of the Doppler broadening, thermal fluctuation, and incoherent scattering noises, our cold atom system with a large OD is a much cleaner model system [19]. For the first time, it allows us to study the transition of photon correlation properties from the biphoton quantum regime (below the threshold) to the MLOPO regime (above the threshold). The experiment agrees with the theory in a large parameter space. Our system provides an ideal platform for studying the quantum interactions of the MLOPO process in a resonant atomic medium.

The experimental setup and relevant atomic energy level diagram are illustrated in Figs. 1(a) and 1(b). We work with ${ }^{85} \mathrm{Rb}$ cold atoms in a dark-line two-dimensional (2D) magneto-optical trap (MOT) [20] with a longitudinal length $L$ of the atomic medium of $1.5 \mathrm{~cm}$. The experiment is run periodically, with each cycle of $1 \mathrm{~ms}$ divided into the MOT time $(0.9 \mathrm{~ms})$ and MLOPO time $(0.1 \mathrm{~ms})$. At end of the MOT time, the atoms are prepared in the ground level $|1\rangle$. In the MLOPO time window, a pump laser $\left(780 \mathrm{~nm}, \omega_{p}\right)$ is blue detuned by $\Delta_{p}$ from the $D 2$ transition $|1\rangle \rightarrow|4\rangle$, and a coupling laser $\left(795 \mathrm{~nm}, \omega_{c}\right)$ is on resonance to the $D 1$ transition $|2\rangle \rightarrow|3\rangle$. The counterpropagating pump and coupling laser beams are collimated with the same $1 / e^{2}$ diameter of $1.40 \mathrm{~mm}$ and aligned with an intersection angle of $\theta=2.8^{\circ}$ to the longitudinal $z$ axis. Phase-matched and 


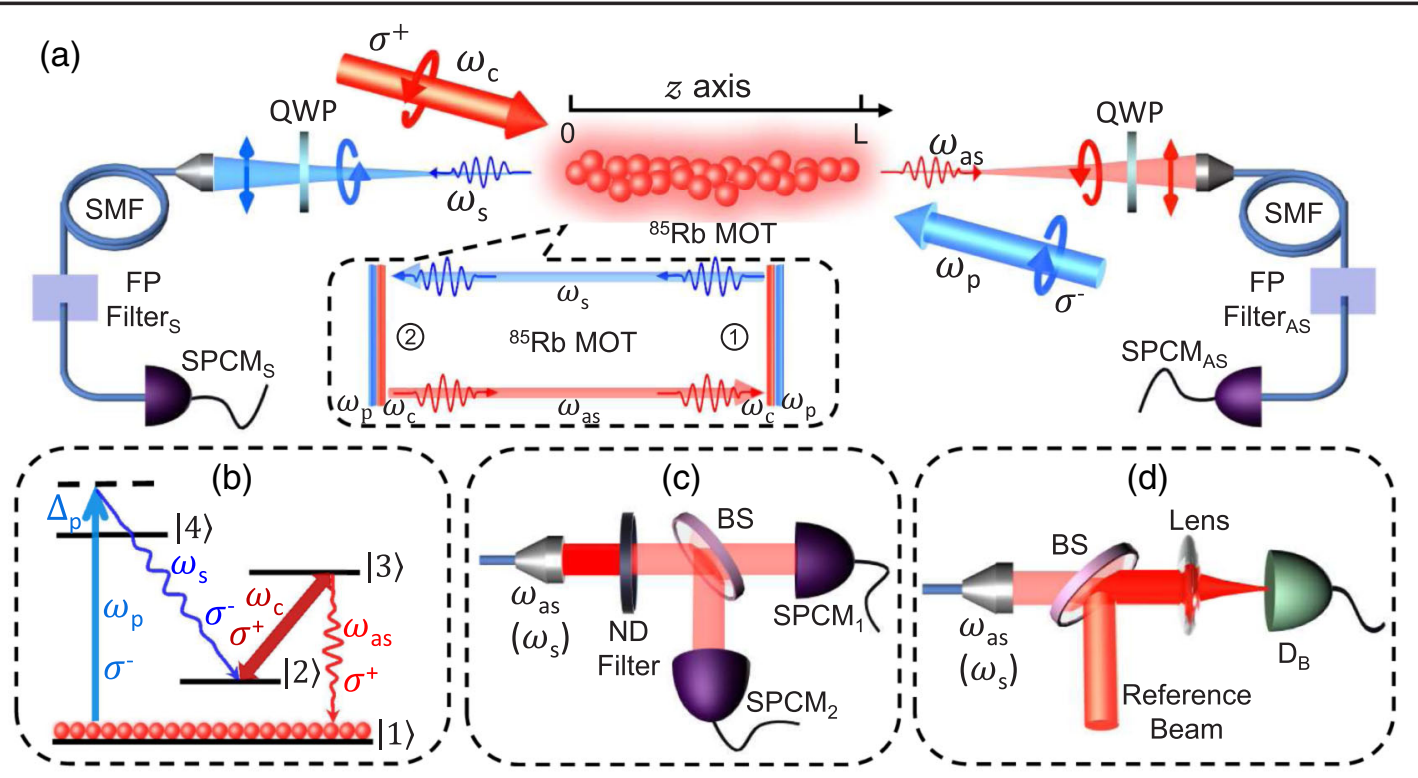

FIG. 1. Experimental configuration for realizing the MLOPO with FWM in cold atoms. (a) Experimental setup. (b) ${ }^{85} \mathrm{Rb}$ atomic energy level diagram and FWM transitions. The atomic hyperfine levels are chosen as $|1\rangle=\left|5 S_{1 / 2}, F=2\right\rangle,|2\rangle=\left|5 S_{1 / 2}, F=3\right\rangle$, $|3\rangle=\left|5 P_{1 / 2}, F=3\right\rangle$, and $|4\rangle=\left|5 P_{3 / 2}, F=3\right\rangle$. The circularly polarized $\left(\sigma^{-}\right)$pump laser (780 nm) is blue detuned by $\Delta_{p}$ from the transition $|1\rangle \rightarrow|4\rangle$, and the coupling laser $\left(\sigma^{+}, 795 \mathrm{~nm}\right)$ is on resonance to the transition $|2\rangle \leftrightarrow|3\rangle$. The generated circularly polarized Stokes $\left(\sigma^{-}\right)$[anti-Stokes $\left(\sigma^{+}\right)$] photons pass through a quarter-wave plate (QWP) and a linear polarizer before they are collected by a SMF. Here the combination of the QWP and polarizer works as a polarization filter. Both Fabry-Perot (FP) filters have the same bandwidth of $500 \mathrm{MHz}$. (c) Detection setup for measuring the autocorrelation function $g^{(2)}(\tau)$, used for spectrum measurement below the threshold. (d) Detection setup for determining the photon spectrum above the threshold by beating the output with a reference laser beam. The reference laser beam beating with the Stokes emission is phase locked to the pump laser. The reference laser beam beating with the anti-Stokes emission is phase locked to the coupling laser.

backward Stokes $\left(\omega_{s}\right)$ and anti-Stokes $\left(\omega_{a s}\right)$ fields are produced in opposing directions along the $z$ axis and collected by two single-mode fibers (SMFs) with a waist diameter of $250 \mu \mathrm{m}$ at the center of the MOT. The emitted Stokes and anti-Stokes photons are then detected by singlephoton counting modules (SPCMs). Figure 1(c) is the detection configuration for measuring the autocorrelation functions which are used to determine the photon spectrum below the threshold. For a stronger emission above the threshold, we use the setup in Fig. 1(d) to measure the spectrum by beating the output to a reference beam. In our experiment, the OD for anti-Stokes photons is 80 , and the dephasing rate $\gamma_{12}$ between the two hyperfine ground states is $2 \pi \times 0.15 \mathrm{MHz}$, which is much smaller than the natural linewidth $\Gamma=2 \pi \times 5.75 \mathrm{MHz}$ of the ${ }^{85} \mathrm{Rb} D 1$ transition.

The inset in Fig. 1(a) shows the mechanism of the nonlinear intrinsic feedback: As a Stokes photon, generated at the right side of the medium (position 1), propagates to the left side (position 2), it induces a FWM process that produces a counterpropagating anti-Stokes photon. As this anti-Stokes photon travels to the right side at position 1, it stimulates another FWM process that generates a second Stokes photon traveling to the left, the same as the first Stokes photon. This feedback results from the FWM nonlinear process and requires no cavity made of mirrors. As the intrinsic feedback meets the amplitude-phase condition, a laserlike oscillation of coherent Stokes and anti-Stokes beams, or MLOPO, will be emitted from the medium. Here the EIT effect not only prevents the antiStokes photons from resonant absorption but also dramatically increases the $\chi^{(3)}$ nonlinearity through resonance enhancement.

To simplify the problem, we use two SMFs to collect generated Stokes and anti-Stokes photons [Fig. 1(a)], so that in this study we focus on the FWM with all four fields in single transverse spatial modes and take the plane-wave approximation for the theoretical treatment. Assuming the pump and coupling beams are undepleted inside the atomic medium and working under the slowly varying amplitude approximation, the counterpropagating single-mode Stokes field envelope $E_{s}\left(\omega_{p}+\omega_{c}-\omega_{a s}, z\right)$ and anti-Stokes field envelope $E_{a s}\left(\omega_{a s}, z\right)$ are governed by the following coupled equations [21]:

$$
\begin{gathered}
\frac{\partial E_{a s}}{\partial z}+\left(\alpha_{a s}-i \frac{\Delta k}{2}\right) E_{a s}=i \kappa_{a s} E_{s}^{*}, \\
\frac{\partial E_{s}^{*}}{\partial z}+\left(g_{s}+i \frac{\Delta k}{2}\right) E_{s}^{*}=i \kappa_{s} E_{a s},
\end{gathered}
$$

where $\alpha_{a s}=-i\left(\omega_{a s} / 2 c\right) \chi_{a s}$ and $g_{s}=-i\left(\omega_{s} / 2 c\right) \chi_{s}^{*}$ describe the linear absorption, gain, and dispersion of the 
anti-Stokes and Stokes fields, with $\chi_{a s}$ and $\chi_{s}$ being their linear complex susceptibilities [22,23]. $\Delta k=\left(k_{a s}-k_{s}\right)-$ $\left(k_{c}-k_{p}\right) \cos \theta$ is the phase mismatching in vacuum. $\kappa_{a s}=\left(\omega_{a s} / 2 c\right) \chi_{a s}^{(3)} E_{p} E_{c}$ and $\kappa_{s}=\left(\omega_{s} / 2 c\right) \chi_{s}^{(3) *} E_{p}^{*} E_{c}^{*}$ are nonlinear coupling coefficients [22]. Solving Eqs. (1) and (2) with the boundary condition $E_{s}(z=L)=0$, we obtain the conversion efficiency between the Stokes and antiStokes fields at the Stokes output port $z=0$ :

$$
\begin{aligned}
\epsilon & =\left|\frac{E_{s}(z=0)}{E_{a s}(z=0)}\right|^{2} \\
& =\left|\frac{2 \kappa_{s}}{q+i \sqrt{4 \kappa_{s} \kappa_{a s}+q^{2}} \cot \left(\frac{L}{2} \sqrt{4 \kappa_{s} \kappa_{a s}+q^{2}}\right)}\right|^{2},
\end{aligned}
$$

where $q=\Delta k-i\left(g_{s}-\alpha_{a s}\right)$ describes the complex phase mismatching in the medium. At the frequencies where the system meets the perfect phase-matching condition, i.e., $q=0$, taking

$$
\kappa_{a s}=\kappa_{s} \frac{\omega_{a s}}{\omega_{s}} \cong \frac{N \sqrt{\sigma_{13} \sigma_{24}} \Gamma}{4\left[\left|\Omega_{c}\right|^{2}+2 \gamma_{12} \Gamma\right]} \frac{\Omega_{p} \Omega_{c}}{\Delta_{p}} \sqrt{\frac{\omega_{a s}}{\omega_{s}}},
$$

we reduce Eq. (3) to

$$
\epsilon=\left|\frac{\sqrt{\omega_{s} / \omega_{a s}}}{\cot \left(\frac{\mathrm{OD} \Gamma \Omega_{p} \Omega_{c}}{4\left[\left[\left.\Omega_{c}\right|^{2}+2 \gamma_{12} \Gamma\right] \Delta_{p}\right.} \sqrt{\frac{\sigma_{24}}{\sigma_{13}}}\right)}\right|^{2} .
$$

Here $\sigma_{i j}$ is the absorption cross section of the transition $|i\rangle \rightarrow|j\rangle$, and $\Omega_{p}$ and $\Omega_{c}$ are the pump and coupling Rabi frequency, respectively. OD $=N \sigma_{13} L$ is the atomic optical depth on the anti-Stokes transition with $N$ as the atomic density. It is clear that the conversion efficiency goes to $\infty$ at

$$
\frac{\mathrm{OD} \Gamma \Omega_{p} \Omega_{c}}{4\left[\left|\Omega_{c}\right|^{2}+2 \gamma_{12} \Gamma\right] \Delta_{p}} \sqrt{\frac{\sigma_{24}}{\sigma_{13}}}=\frac{\pi}{2},
$$

which is the threshold condition for MLOPO. In our system, the dephasing rate $\gamma_{12}$ is very small. With $\gamma_{12} \simeq 0$, Eq. (6) is reduced to

$$
\frac{\Omega_{p}}{\Omega_{c}}=\frac{2 \pi}{\mathrm{OD}} \sqrt{\frac{\sigma_{13}}{\sigma_{24}}} \frac{\Delta p}{\Gamma} .
$$

In such an ideal system, the threshold condition can be met with arbitrarily small pump and coupling powers, as long as the ratio $\Omega_{p} / \Omega_{c}$ satisfies Eq. (7). This unusual behavior agrees with the earlier theoretical prediction in Ref. [18]. For a system with finite $\gamma_{12}$, in order to create the EIT, the coupling power cannot be arbitrarily low. In this case, the coupling power is bounded by $\Omega_{c}^{2} \geq 2 \mathrm{OD} \gamma_{12} \Gamma$. Then the pump power can be determined from Eq. (6).

Figure 2(a) shows typical plots of Stokes and anti-Stokes photon rates as functions of the pump laser power. The

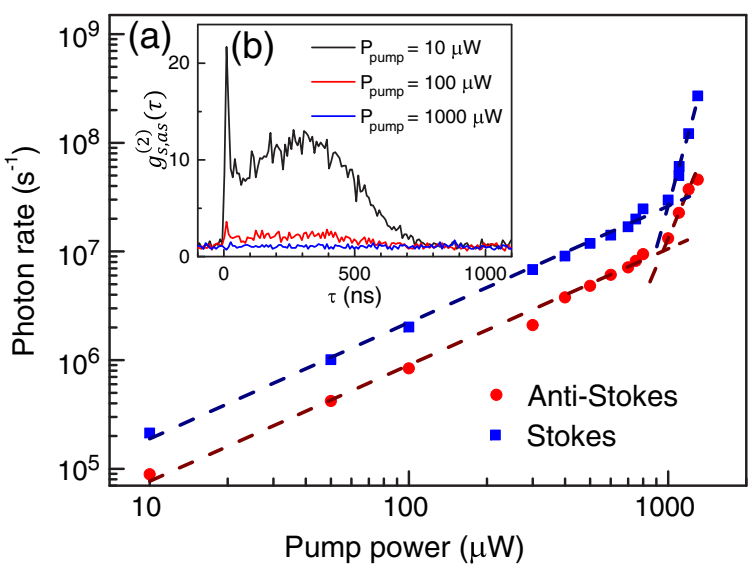

FIG. 2. (a) Output Stokes and anti-Stokes photon rates as functions of the pump laser power, showing the threshold at $1000 \mu \mathrm{W}$. (b) Normalized Stokes-anti-Stokes cross-correlation function under different pump powers. The pump laser detuning is $\Delta_{p}=2 \pi \times 60 \mathrm{MHz}$, and the coupling laser power is $1200 \mu \mathrm{W}$.

pump laser is blue detuned by $60 \mathrm{MHz}$, and the coupling laser power is fixed at $1.2 \mathrm{~mW}$. Both the Stokes and antiStokes plots display a clear signature of the threshold at the pump power of $1000 \mu \mathrm{W}$, showing different slopes below and above the threshold. The low noise cold atom system allows us to study how the Stokes and anti-Stokes normalized two-photon correlation $g_{s, a s}^{(2)}(\tau)=\left\langle\hat{a}_{s}^{+}(t) \hat{a}_{a s}^{+}(t+\right.$ $\left.\tau) \hat{a}_{a s}(t+\tau) \hat{a}_{s}(t)\right\rangle /\left[\left\langle\hat{a}_{s}^{+}(t) \hat{a}_{s}(t)\right\rangle\left\langle\hat{a}_{a s}^{+}(t) \hat{a}_{a s}(t)\right\rangle\right]$ varies in the transition from the nonclassical biphoton regime (below the threshold) to the classical coherent state regime (above the threshold). Here $\hat{a}^{+}$and $\hat{a}$ are the field creation and annihilation operators, respectively. It is well known that two classical fields are bounded to the Cauchy-Schwarz inequality $\left[g_{s, a s}^{(2)}(\tau)\right]^{2} /\left[g_{s, s}^{(2)}(0) g_{a s, a s}^{(2)}(0)\right] \leq 1$ [24]. Figure 2(b) shows $g_{s, a s}^{(2)}(\tau)$ at different pump powers: As we increase the pump laser power to the threshold, the peak value of $g_{s, a s}^{(2)}(\tau)$ reduces to 1 . The peak values of $g_{s, a s}^{(2)}(\tau)$ are 12,2 , and 1 , for the cases with a pump power of 10,100 , and $1000 \mu \mathrm{W}$, respectively. With measured autocorrelation functions $g_{s, s}^{(2)}(0)=g_{a s, a s}^{(2)}(0) \leq 2$ (see Supplemental Material [21]), the case with a pump power of $10 \mu \mathrm{W}$ violates the Cauchy-Schwarz inequality by a factor of 36 , while the case close to the threshold approaches the classical limit.

We next vary the pump laser detuning and coupling laser power to confirm the tunability of the threshold as predicted by the theory [Eqs. (6) and (7)]. Figure 3(a) shows the measured threshold as a function of the pump detuning and coupling power, and Fig. 3(b) shows the pump threshold versus coupling power at different pump detunings. It is clear that, with a fixed pump detuning, the pump threshold is nearly linearly proportional to the coupling power. With a fixed coupling laser power, the pump threshold reduces as we reduce its detuning. The experimental data quantitatively 

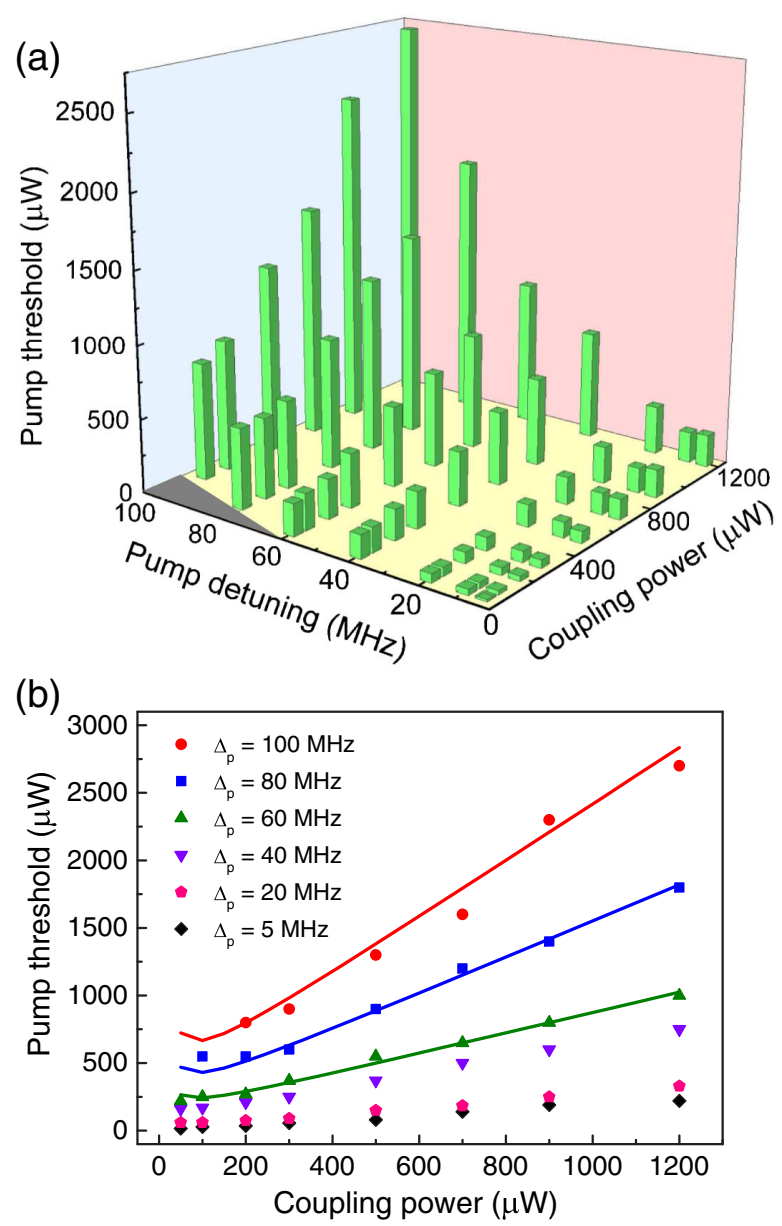

FIG. 3. (a) Pump threshold versus pump detuning and coupling power. (b) Pump threshold as a function of the coupling power at different pump detunings. The dots are experimental data, and the solid lines are calculated from the theory.

agree well with the prediction of Eq. (6) for a large pump detuning $\left(\Delta_{p} \geq 2 \pi \times 60 \mathrm{MHz}\right)$. For a smaller detuning $\left(\Delta_{p}<2 \pi \times 60 \mathrm{MHz}\right)$, while the trend agrees with the theory qualitatively, the exact values deviate from the theoretical prediction, because the absorption of the pump beam makes our undepletion assumption invalid. The lowest pump threshold we achieved in this system is $15 \mu \mathrm{W}$ at the pump detuning of $5 \mathrm{MHz}$ and coupling power of $50 \mu \mathrm{W}$.

We use two different methods to determine the output spectral widths. Below the threshold, we measure the output autocorrelation functions $g_{s, s}^{(2)}(\tau)$ and $g_{a s, a s}^{(2)}(\tau)$ using the setup in Fig. 1(c). Because of the chaotic nature of the emission below the threshold, the first-order coherence function $g^{(1)}(\tau)=\left\langle\hat{a}^{+}(t) \hat{a}_{s}(t+\tau)\right\rangle /\left\langle\hat{a}^{+}(t) \hat{a}(t)\right\rangle$, and the second-order coherence (autocorrelation) function $g^{(2)}(\tau)$ satisfies $g^{(2)}(\tau)=1+\left[g^{(1)}(\tau)\right]^{2}$ [25]. Then from the measured $g^{(2)}(\tau)$ we obtain $g^{(1)}(\tau)$ and thus the emission power spectrum [which is the Fourier transform of $g^{(1)}(\tau)$ ]. In this regime with OD $=80$, the spectral width is determined by both the EIT and the FWM phase-matching

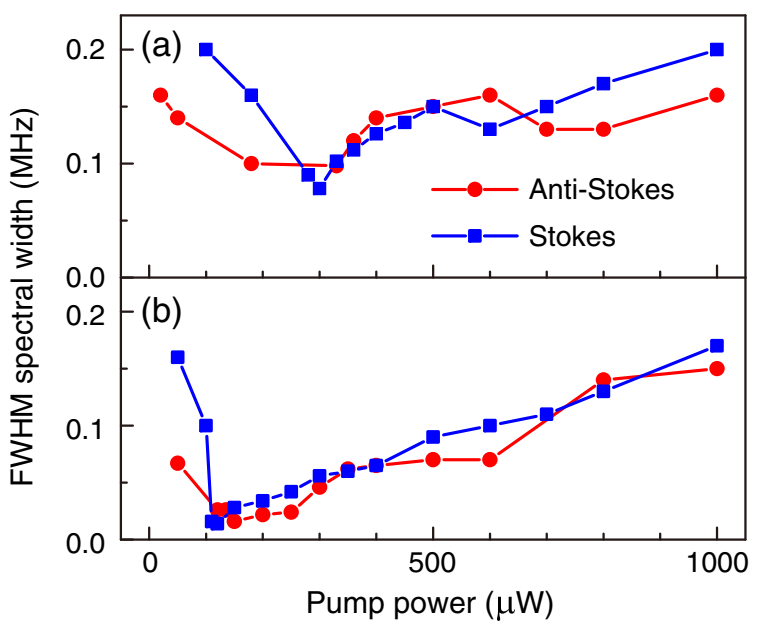

FIG. 4. FWHM spectral widths of the Stokes and anti-Stokes emissions versus the pump power at two different coupling laser powers: (a) 300 and (b) $50 \mu \mathrm{W}$. The pump detuning is $\Delta_{p}=2 \pi \times 60 \mathrm{MHz}$. The pump thresholds for (a) and (b) are about 350 and $200 \mu \mathrm{W}$, respectively.

condition, not by the lifetime of the atomic excited states $|3\rangle$ and $|4\rangle[15,22]$. Above the threshold when the oscillation is formed, the above relation between $g^{(1)}(\tau)$ and $g^{(2)}(\tau)$ is not valid $[25,26]$, and we beat the output with a reference beam to determine the spectrum [Fig. 1(d)]. The measured full width at half maximum (FWHM) of the Stokes and antiStokes output spectrum as functions of the pump power at different coupling laser powers is plotted in Fig. 4. As we increase the pump laser power to its threshold, the output spectral widths reduce significantly. Above the threshold, we observed temporal intensity fluctuations on the outputs which broaden their spectrum gradually as we increase the pump power. The narrowest FWHM spectral width of the MLOPO we achieved is $16 \mathrm{kHz}$ for both outputs, at the pump detuning of $60 \mathrm{MHz}$, with a coupling power of $50 \mu \mathrm{W}$ and pump power of $150 \mu \mathrm{W}$.

In summary, we demonstrate narrow-band MLOPO with a tunable threshold using backward FWM in cold atoms. EIT plays an important role in such a system by not only creating transparency for generated anti-Stokes field but also resonantly enhancing the $\chi^{(3)}$ nonlinearity. The pump threshold can be tuned by varying the operating parameters: the pump detuning, the coupling laser power, and the atomic OD. We achieved a pump threshold as low as $15 \mu \mathrm{W}$, which is limited by our OD and ground-state dephasing. The narrowest FWHM spectral width of the MLOPO we achieved is $16 \mathrm{kHz}$ for both outputs.

This work was supported by the Hong Kong Research Grants Council (Projects No. 601113 and No. 16305615) and in part by the CAS/SAFEA International Partnership Program for Creative Research Teams.

Y. M. and X. G. contributed equally to this work. 
*Corresponding author dusw@ust.hk

[1] S. Noda, Photonic crystal lasers-Ultimate nanolasers and broad-area coherent lasers, J. Opt. Soc. Am. B 27, B1 (2010).

[2] A. Schilke, C. Zimmermann, P. W. Courteille, and W. Guerin, Optical parametric oscillation with distributed feedback in cold atoms, Nat. Photonics 6, 101 (2011).

[3] D.S. Wiersma, The physics and applications of random lasers, Nat. Phys. 4, 359 (2008).

[4] H. Cao, Lasing in random media, Waves Random Media 13, R1 (2003).

[5] S. E. Harris, Proposed backward wave oscillation in the infrared, Appl. Phys. Lett. 9, 114 (1966).

[6] Y. Silberberg and I. Bar-Joseph, Instabilities, Self-Oscillation, and Chaos in a Simple Nonlinear Optical Interaction, Phys. Rev. Lett. 48, 1541 (1982).

[7] A. L. Gaeta, R. W. Boyd, J. R. Ackerhalt, and P. W. Milonni, Instabilities and Chaos in the Polarizations of Counterpropagating Light Fields, Phys. Rev. Lett. 58, 2432 (1987).

[8] G. Khitrova, J. F. Valley, and H. M. Gibbs, Gain-Feedback Approach to Optical Instabilities in Sodium Vapor, Phys. Rev. Lett. 60, 1126 (1988).

[9] J. R. R. Leite, P. Simoneau, D. Bloch, S. L. Boiteux, and M. Ducloy, Continuous-wave phase-conjugate self-oscillation induced by Na-vapour degenerate four-wave mixing with gain, Europhys. Lett. 2, 747 (1986).

[10] M. Pinard, D. Grandclement, and G. Grynberg, Continuouswave self-oscillation using pair production of photons in four-wave mixing in sodium, Europhys. Lett. 2, 755 (1986).

[11] A. Zibrov, M. Lukin, and M. O. Scully, Nondegenerate Parametric Self-Oscillation via Multiwave Mixing in Coherent Atomic Media, Phys. Rev. Lett. 83, 4049 (1999).

[12] C. Canalias and V. Pasiskevicius, Mirrorless optical parametric oscillator, Nat. Photonics 1, 459 (2007).

[13] S. Du, P. Kolchin, C. Belthangady, G. Y. Yin, and S. E. Harris, Subnatural Linewidth Biphotons with Controllable Temporal Length, Phys. Rev. Lett. 100, 183603 (2008).

[14] L. Zhao, X. Guo, C. Liu, Y. Sun, M. M. T. Loy, and S. Du, Photon pairs with coherence time exceeding $1 \mu \mathrm{s}$, Optica 1 , 84 (2014).
[15] L. Zhao, Y. Su, and S. Du, Narrowband biphoton generation in the group delay regime, Phys. Rev. A 93, 033815 (2016).

[16] A. Schilke, C. Zimmermann, and W. Guerin, Photonic properties of one-dimensionally-ordered cold atomic vapors under conditions of electromagnetically induced transparency, Phys. Rev. A 86, 023809 (2012).

[17] J. A. Greenberg and D. J. Gauthier, Steady-state, cavityless, multimode superradiance in a cold vapor, Phys. Rev. A 86, 013823 (2012)

[18] M. Fleischhauer, M. D. Lukin, A. B. Matsko, and M. O. Scully, Threshold and Linewidth of a Mirrorless Parametric Oscillator, Phys. Rev. Lett. 84, 3558 (2000).

[19] B. M. Sparkes, J. Bernu, M. Hosseini, J. Geng, Q. Glorieux, P. A. Altin, P. K. Lam, N. P. Robins, and B. C. Buchler, Gradient echo memory in an ultra-high optical depth cold atomic ensemble, New J. Phys. 15, 085027 (2013).

[20] S. Zhang, J. F. Chen, C. Liu, S. Zhou, M. M. Loy, G. K. Wong, and S. Du, A dark-line two-dimensional magnetooptical trap of $85 \mathrm{Rb}$ atoms with high optical depth, Rev. Sci. Instrum. 83, 073102 (2012).

[21] See Supplemental Material at http://link.aps.org/ supplemental/10.1103/PhysRevLett.119.150406 for the detailed derivation of the coupled nonlinear equations and the measured autocorrelation functions.

[22] S. Du, J. Wen, and M. H. Rubin, Narrowband biphoton generation near atomic resonance, J. Opt. Soc. Am. B 25, C98 (2008).

[23] D. A. Braje, V. Balic, S. Goda, G. Y. Yin, and S. E. Harris, Frequency Mixing Using Electromagnetically Induced Transparency in Cold Atoms, Phys. Rev. Lett. 93, 183601 (2004).

[24] J. F. Clauser, Experimental distinction between the quantum and classical field-theoretic predictions for the photoelectric effect, Phys. Rev. D 9, 853 (1974).

[25] R. Loudon, The Quantum Theory of Light, 3rd ed. (Oxford University, New York, 2000).

[26] For a continuous-wave coherent state, $g^{(2)}(\tau)=1 \neq$ $1+\left[g^{(1)}(\tau)\right]^{2}$. Therefore, above the threshold when the oscillation is formed, we beat the output with a reference beam to determine the spectrum. 